\title{
Commentary \\ Has tamoxifen had its day?
}

Michael Baum

Department of Surgery, University College London, UK

Correspondence: Michael Baum, Consulting Rooms, The Portland Hospital, 212-214 Great Portland St, London, W1W 5QN, UK. Tel: +44 207390 8447; fax: +44 207390 8448; e-mail: m.baum@ucl.ac.uk

Received: 19 June 2002

Breast Cancer Res 2002, 4:213-217 (DOI 10.1186/bcr536)

Revisions requested: 5 July 2002

Revisions received: 16 July 2002

Accepted: 17 July 2002

Published: 27 August 2002

(C) 2002 BioMed Central Ltd

(Print ISSN 1465-5411; Online ISSN 1465-542X)

For a reply to this article see the commentary from V Craig Jordan, http://breast-cancer-research/content/4/6/218

\begin{abstract}
Tamoxifen is probably the most important drug in the history of the management of breast cancer and its development is a tribute to cross talk between laboratory scientists and clinical investigators. Its use as adjuvant therapy has led to a decrease of $20-30 \%$ in age-adjusted cause-specific mortality in the developed world and it is approved in the USA for the chemoprevention of breast cancer in high-risk women. The recent ATAC and IBIS trials have challenged the supremacy of tamoxifen. The present paper is a personal view of the implications for the future use of this drug in competition with the oral aromatase inhibitors. In the opinion of the author tamoxifen will probably remain the mainstay for adjuvant therapy of postmenopausal women with hormone-responsive disease, but maturation of the ATAC data may allow a choice in selected cases. Anastrozole looks like a competitor for the future but we may have to wait another 10 years to find out.
\end{abstract}

Keywords: adjuvant therapy, anastrozole, aromatase inhibitors, chemoprevention, tamoxifen

\section{Introduction}

Tamoxifen has held 'pole position' in the adjuvant treatment of receptor-positive early breast cancer for over 15 years, ever since the first world overview in 1985 [1]. It has also been approved for and widely prescribed in the chemoprevention of breast cancer in the United States since the publication of the P-1 trial [2]. Two recent publications challenge this long-lasting supremacy: namely, the Anastrozole, Tamoxifen Alone or in Combination (ATAC) trial for adjuvant therapy [3], and the IBIS (International Breast Intervention Study) trial for primary prevention [4].

In all modesty, I am in an almost unique position to comment on this challenge. I was the principle investigator of the Nolvadex Adjuvant Trial Organisation [5], which was the first to suggest that tamoxifen had a significant effect on delaying recurrences in the adjuvant treatment of early breast cancer. Jack Cuzick and I were also the first to draw attention to the fact that adjuvant tamoxifen significantly reduced the incidence of contralateral breast cancer [6]. Fifteen to 20 years later, I now find myself as principal investigator of the ATAC trial and a co-author of the IBIS trial, whose principal investigator is my colleague Jack Cuzick.

I therefore believe it is time to reappraise the role of tamoxifen in both the adjuvant and preventive settings, and to seriously consider whether the oral aromatase inhibitors are contenders for the 'gold standard' prize.

\section{The ATAC trial and its implication for adjuvant and chemopreventive regimens}

Although tamoxifen is generally well tolerated and relatively nontoxic, it has become clear during the past decade that prolonged use of this agent is associated with significant gynaecological complications, including prolif- 
erative endometrial abnormalities in postmenopausal women. An increased incidence of endometrial cancer has been reported in association with tamoxifen treatment [7] and the level of risk appears to be time and dose dependent. Most studies involving tamoxifen have found the increased relative risk of developing endometrial cancer while taking tamoxifen to be two to three times higher than that of an age-matched population [8]. The incidence and pattern of this increase in endometrial changes suggest that the underlying mechanism may be related to the oestrogenic properties of tamoxifen. Other side effects related to the oestrogenic properties of tamoxifen include the increased risk of thromboembolic disorders [9].

Most patients who exhibit an initial response to tamoxifen will eventually develop resistance to treatment [10]. Many of these patients, however, respond to both second-line and third-line endocrine therapies, indicating that resistance is not necessarily due to a complete loss of hormone responsiveness. In view of this, alternative agents to tamoxifen are now required to treat breast cancer patients.

The third generation of oral aromatase inhibitors lend themselves ideally as candidates either to enhance the activity of tamoxifen or to replace it entirely in the adjuvant setting.

The ATAC trial in postmenopausal breast cancer patients with early breast cancer is the first report of a new-generation aromatase inhibitor compared with tamoxifen in the early breast cancer setting [3]. An interesting feature of this trial was the inclusion of the combination arm, hence allowing the investigation of any possible additive effects through the use of two drugs with different modes of action. It is possible that anastrozole, by depleting the oestrogen receptor (ER) of its natural ligand while allowing tamoxifen to exert its beneficial effect via saturation of the receptor, could act synergistically in treating early breast cancer.

The ATAC study has been reported in detail [3] but it is worth reviewing in order to clearly understand the message that might have an immediate impact on clinical practice around the world.

Postmenopausal patients with operable invasive breast cancer, who had completed their primary treatment (which included chemotherapy in about $20 \%$ of cases who were judged to be at very high risk of relapse) and who were candidates to receive adjuvant hormonal therapy, were randomized with their informed consent. The patients were randomized to one of three groups: $1.0 \mathrm{mg}$ anastrozole daily plus a placebo for tamoxifen; $20 \mathrm{mg}$ tamoxifen daily plus a placebo for anastrozole; or a combination of the two active agents. A total of 9366 patients from 380 centres in 21 countries were recruited. Five years of treatment were tion for tamoxifen and the wish to remain blinded. We of course have no idea of the optimum duration for anastrozole. It also should be noted that the analysis was triggered by a predetermined number of events when the median duration on therapy was only about 2.5 years.

To understand some of the important subgroup findings, it must be emphasized that the chemotherapy involved those with extensive lymph node involvement but also implied a delay in the start of the allocated endocrine therapy by about 6 months. Furthermore, in many parts of Europe for what was a truly global study, the entry criteria included ER status unknown. However, most of the blocks were retrieved and classified by immunohistochemistry to an ER category. This provided an interesting subgroup.

Disease-free survival was significantly prolonged for patients receiving anastrozole alone compared with those who received either tamoxifen alone (hazard ratio $[\mathrm{HR}]=0.83,0.71-0.96 ; P=0.013)$ or the combination $(\mathrm{HR}=0.81,0.70-0.94 ; P=0.006)$. The combination was not significantly different from tamoxifen alone $(H R=1.02$, $0.89-1.18 ; P=0.8$ ).

When these patients were censored at the time of death, the HR for time to recurrence (including new tumours) was further reduced in the anastrozole arm compared with that of tamoxifen alone $(H R=0.79, \quad 0.67-0.94$; $P=0.008$ ). In comparison with the combination treatment, anastrozole alone also showed a greater benefit for this endpoint ( $\mathrm{HR}=0.75,0.63-0.89 ; P=0.0007)$. No difference was observed, however, between the arm receiving tamoxifen alone and the combination arm $(H R=1.06$, $0.90-1.24 ; P=0.5)$.

As expected, the standard prognostic factors predicted recurrence. The recurrence rate was more than three times higher in hormone receptor-negative women than in those who were hormone receptor-positive.

Two interactions of anastrozole or tamoxifen with potential predictive factors are worthy of comment. The hormone receptor status was close to significance, with the comparison of effects of treatments in the receptor-positive subgroup being predefined in the protocol. In other words, the two drugs were equally ineffective in the ER subgroup.

An interaction, close to significance, within the group receiving chemotherapy first was unexpected and not fully understood. Two possible explanations are being considered: a chance imbalance of key prognostic factors (unlikely), or some unexplained mechanism linked to the delay in starting the endocrine therapy. Alternatively, tamoxifen might be performing better than anticipated because, by the design of the trial, it was provided at the end of chemotherapy rather than concurrently (Intergroup 
trial 0100 presented at the American Society of Clinical Oncology, Orlando, FL, USA, May 2002; principal investigator Kathy Albain). Nevertheless, as we expect tamoxifen to provide added value to chemotherapy, we can predict that anastrozole and tamoxifen are close to equivalent in this setting. This issue requires further events and longer follow-up before any firm conclusions can be drawn on the relative efficacy of anastrozole and tamoxifen after primary chemotherapy treatment.

A striking reduction in contralateral breast primaries as a first event was found in the anastrozole arm of the trial when compared with that of tamoxifen; the odds were reduced by $58 \%$.

Most of the contralateral breast cancers were invasive $(83 \%)$. When the analysis was restricted to these invasive events, the difference was somewhat larger (nine patients in the anastrozole arm versus 30 in the tamoxifen arm versus 23 in the combination arm; odds ratio of anastrozole versus tamoxifen $=0.30,0.14-0.63 ; P=0.0014$ ).

\section{Tolerability of tamoxifen and anastrozole}

There was no distinguishable difference as far as side effects were concerned between tamoxifen alone and the combination. In comparison with tamoxifen alone, however, the profile for anastrozole alone was very different, and in most cases was more beneficial. In particular, there were statistically significant and medically relevant reductions in hot flushes, vaginal discharge, vaginal bleeding, ischaemic cerebrovascular events, venous thromboembolic events and endometrial cancer when receiving anastrozole alone. Endometrial cancers were reduced by $77 \%$ from 13 cases in the tamoxifen arm to three in the anastrozole arm $(P=0.02)$. In contrast, musculoskeletal disorders (i.e. polyarthralgia) and fractures were significantly increased. The greatest increase in fractures on anastrozole treatment appeared to be in the spine, but no increase of hip fractures was seen.

\section{What can we conclude from the ATAC trial?}

Endocrine therapy for breast cancer has enjoyed a remarkable renaissance since the introduction of tamoxifen in the 1970s. It has been estimated that about two-thirds of the observed fall in breast cancer mortality in the UK since the late 1970s can be attributed to this agent [11].

Of course, tamoxifen is not without its problems. Although relatively well tolerated compared with cytotoxic chemotherapy, about 30\% of women on treatment complain of hot flushes, vaginal discharge and vaginal bleeding. Less common, although much more serious, is the long-term risk of endometrial cancer and of thromboembolic disease. It has been estimated that, for every endometrial cancer death, 80 breast cancer deaths have been avoided. Nevertheless, fear of this complication prompts transvaginal ultrasound scans and hysteroscopy in a large number of those women complaining of gynaecological symptoms, placing additional pressure on overstretched services.

Anastrozole shows superior efficacy to tamoxifen at this relatively early stage of follow-up, with a $17 \%$ relative risk reduction in disease-free survival in the intention-to-treat population and a 19\% improvement when compared with the combination arm. Although statistically significant and impressive in relative terms, this still only translates into an absolute improvement in disease-free survival of $2-3 \%$. It is of course too early to be able to describe outcomes in terms of all-cause and cause-specific mortality, bearing in mind that the median duration of therapy was only about 2.5 years out of the intended 5 years of treatment. It also needs to be noted that a disproportionate number of early events where among the less informative HR-negative and HR-unknown groups.

The most surprising result was for the combination group, which fared no better than tamoxifen alone and fared significantly worse than anastrozole alone. One possible explanation is that in an oestrogen-deprived environment tamoxifen is 'seen' as an agonist, whereas in a normal oestrogen-rich environment tamoxifen can exert its classical anti-oestrogen effect. Support for this viewpoint comes from the nude mouse xenograft experiments [12].

Although a rare event, the fear of endometrial cancer associated with exposure to tamoxifen means that most of those women with gynaecological symptoms might have been subjected to invasive investigations. This advantage for anastrozole could, in the long term, save much unnecessary anxiety and health service costs.

The disadvantages of anastrozole compared with tamoxifen are found in the contrast between women with chronic oestrogen deprivation compared with women on a drug with a weak agonist effect. This can already be noted in the modest but significant excess of fractures already observed in the anastrozole group. Whether this is a result of the protective effect of tamoxifen on bone compared with an untreated population, or whether it is due to oestrogen deprivation because of aromatase inhibition, is at this stage speculative. In pragmatic terms, of course, it does not matter what the mechanism is because women will have to make an informed choice between one drug or the other; no treatment is not an option except in cases with an extremely good prognosis.

Nevertheless, excess fractures are a side effect that can be managed if anticipated. Clinicians opting to start women on anastrozole should request a baseline bone density scan, which should be repeated at perhaps 12month intervals. Women whose bone density starts to fall 
into the osteopaenic range could be considered for a variety of options. For example, stopping anastrozole if they have been on treatment for more than 3 years (bearing in mind the relatively small difference in absolute benefit between 2 and 5 years of therapy) or starting on a bisphosphonate, which as well as protecting the skeleton might even reduce the risk of skeletal metastases [13].

Perhaps the most impressive result of the ATAC trial at this stage is the near $60 \%$ reduction in the incidence of contralateral invasive cancers for anastrozole treatment compared with tamoxifen treatment. Yet tamoxifen has been known to be associated with a significant reduction in contralateral disease compared with a control population since 1985 [1]. In the latest overview, 5 years of tamoxifen is associated with a $50 \%$ reduction in the contralateral breast cancer [14]. These observations have been translated into a prevention setting for high-risk women in the National Surgical Adjuvant Breast and Bowel Project P-1 study [2].

If these trends in the incidence of contralateral breast cancers persist, then anastrozole has the potential to prevent (or delay) up to $80 \%$ of all cancers. These observations have already been acted on in the design of the second UK/ANZ prevention trial, IBIS II (J Cuzick, personal communication).

\section{The IBIS trial and the future of tamoxifen as a chemopreventive agent}

Three clinical trials have reported on the use of tamoxifen to prevent breast cancer, with mixed results $[2,15,16]$. The overall evidence supports a reduction in the risk of developing breast cancer, but it is unclear whether this benefit outweighs the risks and side effects associated with tamoxifen.

The IBIS trial, which has just been reported [4], in my mind clarifies the issue beyond doubt. This was a double-blind placebo-controlled trial conducted in 7140 women at increased risk of breast cancer, aged 35-70 years. The primary outcome measure was breast cancer incidence (including ductal carcinoma in situ). Other cancers, vascular disease, side effects and mortality were secondary endpoints.

A total of 169 breast cancers were observed in both arms (tamoxifen versus placebo) combined. There was a 33\% (Cl 9-51) reduction in breast cancer incidence associated with tamoxifen compared with placebo (68 versus 101 cases, $P=0.01)$. Endometrial cancer was increased about twofold (11 versus five cases) but failed to reach conventional levels of significance $(P=0.2)$. Thromboembolic events were substantially increased (odds ratio $=$ 2.6, $\mathrm{Cl} 1.7-4.2, P<0.0001)$. Vasomotor and gynaecologic symptoms were increased, but benign breast com- excess of deaths in the tamoxifen arm compared with placebo (25 patients versus 10 patients, $P=0.016$ ). This was primarily due to an excess of cardiac and vascular deaths.

The increased incidence of gynaecologic problems, particularly the increased requirement for hysterectomy and oophorectomy, is of some concern. These may be a result of an increased incidence of vaginal bleeding, of endometrial polyps, of endometrial thickening or of ovarian cysts. All these gynaecological sequelae of tamoxifen and their investigation must have a profound negative impact on the quality of life of otherwise well women.

The relatively low breast cancer death rate highlights the importance of a full evaluation of side effects. The excess mortality in the tamoxifen arm seen in the IBIS trial may in part be due to statistical variability. However, the excess of thromboembolic events seen in all trials and the smaller excess of thromboembolic deaths seen in the IBIS trial and the P-1 study do indicate that this is the most important complication of tamoxifen use, and that every effort should be taken to reduce this risk.

While it is clear that tamoxifen can reduce the incidence of ER-positive breast cancer, the overall risk/benefit ratio for tamoxifen in the preventive setting is still unclear at present. Further long-term follow-up study of breast cancer incidence and mortality, other causes of death and side effects in the ongoing trials remain essential. For the time being, tamoxifen for the prevention of breast cancer has to be considered either experimental or 'with a great future behind it'. I am clearly not alone in this belief as the steering committee designing the IBIS II trial have dropped tamoxifen as the control in favour of a placebo to compare with anastrozole (J Cuzick, personal communication).

\section{Conclusions}

Although one of the first to propose it, I no longer believe that tamoxifen has a role in the chemoprevention of breast cancer. I think what has happened in America in this regard is a great mistake. I believe that the P-1 study was presented too early and its follow-up was aborted too soon. For this reason, the currently recruiting STAR trial comparing tamoxifen with raloxifene will be uninterpretable, for want of a placebo control group, in spite of its astronomical investment in dollars and volunteers [17].

I believe it is premature to abandon tamoxifen in the adjuvant setting but at least there is a real choice for the first time in nearly 20 years. For example, in my opinion, elderly women with a relatively short life expectancy because of comorbidity, with or without an increased risk for thromboembolic disease, would be better off on anastrozole than tamoxifen. As for the rest, I predict that a further year or two of follow-up in the ATAC trial should resolve the issue. 
In the meantime the ATAC steering committee has agreed to close down the combined arm but continue the monotherapy arms blinded within the study. Tamoxifen may not have had long enough to express its full potential and we remain concerned about the long-term effects of aromatase inhibitors on bone mineral metabolism.

Once we've sorted out the ethical issues concerning the archival material in this study, our pathology sub committee, chaired by Prof. Mitch Dowsett, will begin work on the microarrays to allow us to search for predictive factors of response. In particular we are interested to find out if overexpression of ErbB-2 results in a preferential benefit from anastrozole, and we can therefore be selective in our choice of adjuvant therapy according to the specific tumour phenotype. What an historical step forward that would be? However, until that golden day dawns I will hedge my bets, breast cancer that enigmatic disease, will never cease to surprise us.

\section{References}

1. Early Breast Cancer Trialists' Collaborative Group: Effects of adjuvant tamoxifen and of cytotoxic therapy on mortality in early breast cancer. An overview of 61 randomized trials among 28,896 women. $N$ Engl J Med 1988, 319:1681-1692.

2. Fisher B, Costantino JP, Wickerham DL, Redmond CK, Kavanah M, Cronin WM, Vogel V, Robidoux A, Dimitrov N, Atkins J, Daly M, Wieand S, Tan-Chiu E, Ford L, Wolmark N: Tamoxifen for prevention of breast cancer: report of the National Surgical Adjuvant Breast and Bowel Project P-1 study. J Natl Cancer Inst 1998, 90:1371-1387.

3. The ATAC Trialist Group: Anastrozole alone or in combination with tamoxifen versus tamoxifen alone for adjuvant treatment of postmenopausal women with early breast cancer: first results of the ATAC randomised trial. Lancet 2002, 359:21312139.

4. IBIS Working Party: First results from the IBIS-I breast cancer prevention trial. Lancet (in press).

5. Nolvadex Adjuvant Trial Organisation: Controlled trial of tamoxifen as adjuvant agent in management of early breast cancer. Lancet 1983; i:257-261.

6. Cuzick J, Baum M: Tamoxifen and contralateral breast cancer [letter]. Lancet 1985, 2:282.

7. Sismondi P, Biglia N, Volpi E, Giai M, de Grandis T: Tamoxifen and endometrial cancer. Ann NY Acad Sci 1994, 734:310-321.

8. Bergman L, Beelan MLR, Gallee MPW, Hollema H, Benraadt J, van Leeuwen FE: Risk and prognosis of endometrial cancer after tamoxifen for breast cancer. Lancet 2000, 356:881-888.

9. Cutuli B, Petit JC, Fricker JP, Jung GM, Schumacher C, Velten M, et al:: Adjuvant tamoxifen in breast cancer treatment in postmenopausal women: occurrence of thromboembolic complications. Oncol Rep 1994, 1:59-63.

10. Howell $A$, Dowsett M: Recent advances in endocrine therapy of breast cancer. Br Med J 1997, 315:863-866.

11. Brown P: UK death rates from breast cancer fall by a third. BMJ 2000, 321:849.

12. Gottardis MM, Jordan VC: Development of tamoxifen stimulated growth of MCF-7 tumours in athymic mice after longterm anti-oestrogen administration. Cancer Res 1988, 48: 5183-5187.

13. Van Poznak C: How are bisphosphonates used today in breast cancer clinical practice? Semin Oncol 2001, 28(suppl 11):69-74.

14. Early Breast Cancer Trialists' Collaborative Group: Tamoxifen for early breast cancer: an overview of the randomised trials. Lancet 1998, 351:1451-1467.

15. Powles T, Eeles R, Ashley S, Easton D, Chang J, Dowsett M, Tidy A, Viggers J, Davey J: Interim analysis of the incidence of breast cancer in the Royal Marsden Hospital tamoxifen randomised chemoprevention trial. Lancet 1998, 352:98-101.
16. Veronesi U, Maisonneuve P, Costa A, Sacchini V, Maltoni C, Robertson C, Rotmensz N, Boyle P: Prevention of breast cancer with tamoxifen: preliminary findings from the Italian randomised trial among hysterectomised women. Lancet 1998, 352:93-97.

17. Cauley JA, Norton L, Lippman ME, Eckert S, Krueger KA, Purdie DW, Farrerons J, Karasik A, Mellstrom D, Ng KW, Stepan JJ, Powles TJ, Morrow M, Costa A, Silfen SL, Walls EL, Schmitt H, Muchmore DB, Jordan VC, Ste-Marie LG: Continued breast cancer risk reduction in postmenopausal women treated with raloxifene: 4-year results from the MORE trial. Breast Cancer Res Treat 2001, 65:125-134. 